\title{
The next move
}

DOI:

10.1038/nrc1988

\section{URLs \\ EGFR}

http://www.ncbi.nlm.nih.gov/ entrez/query.fcgi?db=gene\&c $\mathrm{md}=$ RetrieveEdopt=full_ report\&list_uids=1956

ERBB2

http://www.ncbi.nlm.nih.gov/ entrez/query.fcgi?db=gene\&c $\mathrm{md}=$ Retrieve\&dopt=full report\&list_uids=2064

ADAM17

http://www.ncbi.nlm.nih.gov/ entrez/query.fcgi?db=gene\&c $\mathrm{md}=$ RetrieveEdopt=full reportElist_uids $=6868$

NSCLC

http://www.cancer.gov/ cancertopics/types/lung/
Mutations in the epidermal growth factor receptor (EGFR, also known as ERBB1) that result in resistance to the EGFR inhibitor gefitinib have prompted closer examination of EGFR in patients with non-small-cell lung cancer (NSCLC). Changes in the dimerization partners of EGFR, such as ERBB2 (also known as HER2), or triggering alternative ErbB signalling pathways, such as ERBB3 (also known as HER3), have a role in gefitinib resistance. Two groups have investigated the next move to circumvent this resistance - Carlos Arteaga and colleagues show that patients with intragenic mutations in the kinase domain of ERBB2 are sensitive to ERBB2 inhibitors, and Bin-Bing Zhou and colleagues show that the inhibition of an upstream activator of the ligand for ERBB3, ADAM17, causes the death of certain gefitinib-resistant NSCLC cells.

About $2 \%$ of patients with NSCLC have intragenic mutations in ERBB2, which is a preferred dimerization partner of EGFR and leads to the activation of ErbB signalling pathways. But how does this mutation affect tumour cells? When Arteaga and colleagues transfected human immortalized bronchial or mammary epithelial cell lines with the ERBB2 mutant (encoding ERBB2 ${ }^{\mathrm{YVMA}}$ ) or wild-type $E R B B 2$, only cells with the mutant receptor formed tumours in nude mice. Cells transfected with the gene encoding ERBB2 $2^{\mathrm{WT}}$ or ERBB2 ${ }^{\mathrm{YVMA}}$ and treated with a bivalent ligand to induce receptor homodimerization showed that ERBB2 ${ }^{\mathrm{YVMA}}$ phosphorylation - which was high even in the absence of ligand - was more inducible than ERBB2 ${ }^{\mathrm{WT}}$. In addition, basal levels of phosphorylation and, therefore, activation of the signalling molecules EGFR, SRC, AKT and MAPK were markedly higher in ERBB2 ${ }^{\text {YVMA }}$ cells. Treatment with gefitinib efficiently blocked ligandinduced target phosphorylation in the cells that expressed ERBB2 ${ }^{\text {WT }}$, probably through interference with EGFR-ERBB2 crosstalk. However, the inhibition of EGFR by gefitinib had no affect on the constitutively active ERBB2 mutant or on its ability to engage with and activate ErbB signal transducers.

So, could this resistance be circumvented by inhibiting ERBB2 rather than EGFR? Arteaga and colleagues found that the ERBB2 antibody trastuzumab inhibited the growth and invasion of cells that expressed the ERBB2 mutant. In addition, treatment with the EGFR and ERBB2 dual inhibitors lapatinib or CI-1033 inhibited growth and invasion of both wild-type and mutant cells. The combination of lapatinib and trastuzumab was more potent than either drug alone in suppressing the growth of mutant cells. Knockdown of mutant ERBB2 also resulted in the inhibition of cell proliferation and cell death, and restored sensitivity to gefitinib in lung cancer cells.

Zhou et al. examined the activation of an alternative ErbB pathway, ERBB3 (also known as HER3), as a possible mechanism of resistance to gefitinib. The only known ligand for ERBB3 is heregulin, and this needs be to proteolytically cleaved to become active. ADAM17 is a metalloprotease that is thought to mediate this cleavage. The authors found that many primary tumours from NSCLC patients that they examined had activated ERBB3, co-expressed heregulin and ERBB3, and had increased expression of ADAM17. Heregulin-induced ERRB3 activation in NSCLC cell lines caused resistance to gefitinib. RNA interference of ADAM17 or treatment with a smallmolecule inhibitor of ADAM 17, INCB3619, prevented the cleavage of heregulin and abrogated heregulin-dependent ERBB3 signalling in A549 NSCLC cells. Furthermore, the INCB3619-treated A549 cells were then resensitized to treatment with gefitinib.

So, both these reports have increased our understanding of the ErbB signalling pathways and the approaches explored hold promise for overcoming resistance to gefitinib in NSCLC.

Ezzie Hutchinson

ORIGINAL RESEARCH PAPERS Wang, S. E. et al. ERBB2 kinase domain mutation results in constitutive phosphorylation and activation of ERBB2 and EGFR and resistance to EGFR tyrosine kinase inhibitors. Cancer Cell 10, 25-38 (2006) | Zhou, B.-B. S. et al. Targeting ADAM-mediated ligand cleavage to inhibit HER3 and EGFR pathways in non-small cell lung cancer. Cancer Cell 10,39-50 (2006)

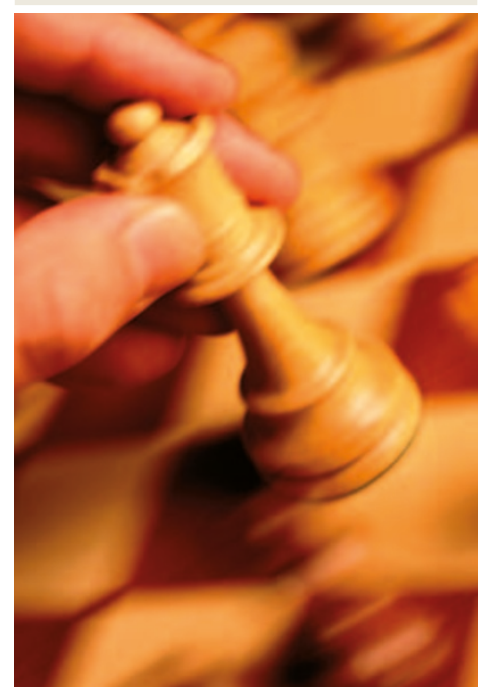

